\title{
Getting a THUMP from a WIMP
}

\author{
Hooman Davoudiasl and Gopolang Mohlabeng \\ Physics Department, Brookhaven National Laboratory, \\ Upton, New York 11973, U.S.A. \\ E-mail: hooman@bnl.gov, gmohlabeng@bnl.gov
}

ABSTRACT: Producing an acceptable thermal relic abundance of dark matter with masses $\gg 10^{2} \mathrm{TeV}$ is a challenge. We propose a novel mechanism where GeV-scale states establish a tiny thermal relic abundance for dark matter, which is later promoted to ultra massive status by a very light scalar. We refer to this dark matter as a THermal Ultra Massive Particle (THUMP). Direct detection of THUMPs can be naturally expected due to large scattering cross sections mediated by low mass states that couple THUMPs to the Standard Model. Our model generically leads to signals for the associated $\mathrm{GeV}$-scale states at accelerator experiments.

Keywords: Beyond Standard Model, Cosmology of Theories beyond the SM

ARXIV EPRINT: 1912.05572 


\section{Contents}

1 Introduction 1

2 A specific model 2

3 Relic abundance in the early Universe $\quad 3$

$\begin{array}{lll}4 & \text { Constraints and phenomenology } & 7\end{array}$

5 Discussion and conclusions $\quad 9$

\section{Introduction}

The search for clues to the identity of dark matter (DM) continues. So far, no experiment has uncovered conclusive evidence regarding the properties of DM and the range of possibilities for what it could be remains vast. In this situation, one may be compelled to try and look for potential candidates wherever possible. However, launching a search in every possible direction is not feasible and very often one needs some motivating factor to justify the effort. Here, theoretical considerations could point to promising targets.

The weak scale has been, for a long time, considered a likely place for new physics to emerge, in large part due to arguments based on a natural Higgs sector for the Standard Model (SM). This scale is also typical for production of thermal relic DM, a fact that has strongly motivated searches for DM particles of corresponding mass. However, after decades of dedicated searches for both new weak scale particles and DM at accelerators and direct detection experiments, no conclusive evidence of either has been uncovered. While this may point to the elusiveness of such new states or the limitations of current experimental techniques, one could also start to entertain new or less examined possibilities.

Apart from the well-established weak scale, the scale of a possible quantum theory of gravity is another conceptually plausible place where new phenomena may arise. This scale is taken to be set by the Planck mass $M_{\mathrm{Pl}} \approx 1.2 \times 10^{19} \mathrm{GeV}$, which is also typically assumed to mark the end of short distance physics. Between the weak and Planck scales there could be several other motivated scales where new phenomena may appear. For example, unification of known forces of Nature in a Grand Unified Theory often points to masses $\gtrsim 10^{15} \mathrm{GeV}$ for new states. Also, the strong CP problem of QCD can be elegantly addressed through the Peccei-Quinn mechanism which typically points to scales $f_{P Q} \lesssim 10^{12} \mathrm{GeV}$, to avoid conflict with cosmology ("overclosing" the Universe) [1-3]. However, astrophysical constraints on the associated axion demand $f_{P Q} \gtrsim 10^{9} \mathrm{GeV}[4]$. So, $10^{9} \mathrm{GeV} \lesssim f_{P Q} \lesssim$ $10^{12} \mathrm{GeV}$ provides another motivated window at very high scales (the axion could be a good DM candidate at the upper end of the window). 
Although the above high scales provide motivation to consider DM masses well above the weak scale, DM relic abundance is generally assumed to be non-thermal for masses above $\sim 100 \mathrm{TeV}[5]$. In principle, one could construct such models of ultra massive DM, but generically the underlying physics and the signals of this DM may not be accessible, due to the high scales involved [6-9]. Different mechanisms for setting the thermal relic abundance of ultra massive DM, leading to various astrophysical signatures have been considered in the literature, see for example [10-13]. It would be interesting to consider possible mechanisms for setting the thermal relic abundance of ultra massive DM that would naturally lead to accessible signals in direct detection experiments or at accelerators. Such a scenario may be motivated by possible multi-scatter [14-17] or single scatter signals in current or near future detectors.

In this work, we examine whether ultra massive DM particles could be produced initially as a thermal relic, but with a much smaller mass. This would suggest that the thermal relic abundance is set to very small values, which will yield the correct energy density once DM attains a large mass later. We propose a scenario in which the late time slow-roll of an ultra-light modulus scalar field drives the DM mass to values $\gg 100 \mathrm{TeV}$. We note here that similar scenarios were constructed in refs. [18, 19], but with different phenomenology and physics outcomes.

Intuitively, the small relic abundance implies very large annihilation cross sections. We will illustrate through a simple model that this typically points to an initial DM mass $\lesssim \mathrm{GeV}$ and associated dark states that mediate "secluded" annihilations. However, once the DM becomes very heavy, it maintains its strong coupling to light mediators which would naturally lead to detectable signals in the laboratory, such as through direct detection of $\mathrm{DM}$ or searches for the mediators in accelerator experiments. We will refer to our proposed DM candidate as a THermal Ultra Massive Particle (THUMP).

In the following sections, we outline a simple model that realizes the above scenario using a Dirac fermion for DM and a light (sub-GeV) vector particle, which we take to be a "dark photon" coupled to the SM through kinetic mixing. One could invoke a late phase transition (For very recent work in this direction, see refs. [20-22]) or a very light modulus that effects the mass variation of the DM particle from light ( $\mathrm{GeV}$ scale) to very heavy. Here, we will focus on the second possibility, which will typically lead to a long range force acting on THUMPs and could possibly offer further astrophysical signatures.

\section{A specific model}

Let us first consider a DM particle $\chi$ with mass $m_{\chi}^{i}$ in the early Universe, i.e. before Big Bang Nucleosynthesis (BBN). Here, $\chi$ is assumed to have charge $Q_{D}$ under a dark $U(1)^{\prime}$ interaction mediated by $A_{\mu}^{\prime}$ of mass $m_{A^{\prime}} \lesssim m_{\chi}^{i}$. We also suppose that the DM $\chi$ interacts with a very light scalar $\phi$. The Lagrangian for these interactions is given by

$$
-\mathcal{L} \supset\left(\lambda \phi+m_{\chi}^{i}\right) \bar{\chi} \chi-i g_{D} Q_{D} A_{\mu}^{\prime} \bar{\chi} \gamma^{\mu} \chi+\frac{1}{2} m_{A^{\prime}}^{2} A^{\prime 2}+\frac{1}{2} m_{\phi}^{2}\left(\phi-\phi_{0}\right)^{2} .
$$

In eq. (2.1), $\phi$ sources a large mass $m_{\chi}^{f} \gg m_{\chi}^{i}$ for the DM once it starts evolving in its potential. This mass is given by the vacuum expectation value (vev) $\phi=\phi_{0}$ which yields 


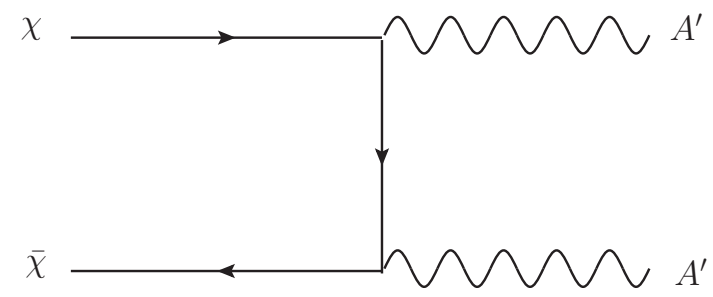

Figure 1. Annihilation of DM $\chi$ into "dark photon" pairs, which later decay into SM states (only $t$-channel shown).

$m_{\chi}^{f}=m_{\chi}^{i}+\lambda \phi_{0}$. The idea here is that dark matter freeze-out is governed by $m_{\chi}^{i}$ with $\phi$ frozen near $\phi=0$, leading to an efficient annihilation; we will later discuss how this could be arranged due to thermal effects in our scenario. During this time, the thermal relic abundance of DM is set by the annihilation of $\chi$ into pairs of $A^{\prime}$ through a large coupling $g_{D} Q_{D}$ (represented by figure 1 ). The vector $A^{\prime}$ then decays into SM particles before BBN; this is thus a secluded annihilation scenario [23]. The coupling of $A^{\prime}$ to the SM will be assumed to result from kinetic mixing between $U(1)^{\prime}$ and SM hypercharge $\mathrm{U}(1)_{Y}$ [24]:

$$
\frac{\varepsilon}{2 \cos \theta_{W}} F_{\mu \nu}^{\prime} F_{Y}^{\mu \nu}
$$

where $\varepsilon \ll 1$ is the kinetic mixing parameter, $\theta_{W}$ is the weak mixing angle, and $F_{\mu \nu}$ refers to the associated field strength tensor for each U(1) interaction. For the rest of this discussion, we set $Q_{D}=1$.

After DM has frozen out and the relic abundance has been set, the $\phi$ field rolls and oscillates, where its expectation value increases the DM mass to $m_{\chi}^{f}$. Since $m_{A^{\prime}} \lesssim m_{\chi}^{i}$, the annihilation represented by figure 1 is very efficient, especially for $g_{D} \gtrsim 1$, resulting in a small relic abundance in the early Universe. The DM energy density at late times is given by $\rho_{\chi}=m_{\chi}^{f} n_{\chi}$ and the relic abundance is given by $\Omega_{\chi} h^{2}=\rho_{\chi} h^{2} / \rho_{c}$, where $h=0.678(9)$ is the Hubble expansion rate scale [4] and $\rho_{c} \approx 1.05 \times 10^{-5} h^{2} \mathrm{GeV} / \mathrm{cm}^{3}$ is the critical cosmic energy density. With the rolling of $\phi$ after freeze out, raising the mass of $\chi$ to $m_{\chi}^{f}$, we obtain the DM relic energy density we expect today. We will next estimate the typical parameters that could give rise to the above qualitatively described scenario.

\section{Relic abundance in the early Universe}

In this work we consider only $\chi$ as the DM and not $\phi$. However in principle $\phi$ could contribute to the DM budget in the Universe, depending on its mass and when it starts oscillating. As indicated in eq. 2.1, the potential for $\phi$ is given by $V(\phi) \sim m_{\phi}^{2}\left(\phi-\phi_{0}\right)^{2}$. At early times when $H>m_{\phi}$, the $\phi$ field is overdamped and does not oscillate up until $H \lesssim m_{\phi}$. Since we are interested in DM number densities that are far smaller than the usual thermal relic values, the freeze out parameter $x_{f} \gtrsim 20$. Here $x_{f} \equiv m_{\chi} / T_{f}$, where $T_{f}$ is the freeze out temperature. Given that the large annihilation cross sections required will lead us to $m_{\chi} \lesssim \mathrm{GeV}$, we expect that $T_{f}$ is $\mathcal{O}(10 \mathrm{MeV})$, larger than $\sim \mathrm{MeV}$, to avoid 
disrupting BBN. Therefore, a typical expectation in our scenario is that the freeze out Hubble scale $H_{f} \sim T_{f}^{2} / M_{\mathrm{Pl}} \sim 10^{-14} \mathrm{eV}$, as a rough guide.

In what follows, we will consider final masses for DM up to $m_{\chi}^{f} \sim 10^{9} \mathrm{GeV}$. We find that pushing beyond this limit could require dealing with non-perturbative effects that could complicate the analysis and render our estimates unreliable. However, there should be no obstacle, in principle, to push beyond the above mass. We note that the final value of the scalar field $\phi_{0}$ could be much larger than $\sim 10^{9} \mathrm{GeV}$, as long as $\lambda \ll 1$.

Let us consider $\phi_{0} \sim 10^{15} \mathrm{GeV}$, as may be expected in an ultraviolet (UV) framework, corresponding to $\lambda \sim 10^{-6}$. If $m_{\phi} \lesssim 10^{-14} \mathrm{eV}$, then $\phi$ will start to roll after the freeze out era discussed earlier, corresponding to typical temperatures $\sim 10 \mathrm{MeV}$. Since we are assuming that the initial value of $\phi$ is given by $\phi_{i} \approx 0$, the energy density initially stored in $\phi$ will be given by $m_{\phi}^{2} \phi_{0}^{2} \lesssim 10^{20} \mathrm{eV}^{4}$. Absent any significant interactions, this energy density would redshift by $\left(T_{\text {eq }} / T_{f}\right)^{3} \sim 10^{-21}$, where $T_{\text {eq }} \sim 1 \mathrm{eV}$ marks the era of matterradiation equality, with energy density $\mathcal{O}\left(T_{\mathrm{eq}}^{4}\right)$. Hence, with the above sample values of parameters, it seems that if $\phi$ attains its ground state instantaneously, it does not have enough energy to account for the final energy stored in DM. Below, we will show that this apparent mismatch can be accounted for once the interactions of $\phi$ with the relic $\chi$ particles is properly considered. ${ }^{1}$ We also note that the above initial energy density stored in $\phi$ is much less than the radiation energy density of $\sim(10 \mathrm{MeV})^{4}$, so the initial potential energy of $\phi$ does not lead to inflation.

For the above scenario to yield initially small $\chi$ mass, it is required that $\phi_{i} \approx 0$ to begin with. One can just assume that this is the case, but it would be more interesting if the underlying physics could lead to such an initial condition. Here, we argue that this is indeed the case in our model (see also ref. [18]). Let us declare upfront that we will ignore possible quantum corrections to the potential for $\phi$. This question may need to be addressed in a fuller theory describing the above effective interactions and could require new dynamics beyond what has been considered here. While we do not claim a rigorous connection to the Higgs hierarchy problem in the SM, we simply note that the seeming insular nature of the SM could be hinting at a more subtle effect for quantum contributions to scalar masses. We do, however, consider the effect of the ambient medium on scalar masses.

For the assumed interactions, the thermal effects of the DM on the scalar $\phi$ can be parametrized by $\delta m_{\phi}^{2} \sim \lambda^{2} T^{2},[25]$ where $T \gtrsim 10 \mathrm{MeV}$ and $\lambda \sim 10^{-6}$. Hence, the mass of $\phi$, considering thermal corrections will be given by

$$
m_{\phi}^{2}(T) \sim m_{\phi}^{2}+\lambda^{2} T^{2}
$$

where we have left out $\mathcal{O}(1)$ factors that would not affect our main conclusions. With the above contribution, the temperature dependent value of the scalar vev will be given by

$$
\phi(T) \sim \frac{m_{\phi}^{2} \phi_{0}}{m_{\phi}^{2}+\lambda^{2} T^{2}} .
$$

\footnotetext{
${ }^{1}$ We thank Yue Zhao for discussions on this point.
} 
For typical values of parameters, $m_{\phi} \sim 10^{-14} \mathrm{eV}, \lambda \sim 10^{-6}$, and $T \sim \mathcal{O}(10 \mathrm{MeV})$, we see that $\phi(T) \sim 10^{-30} \phi_{0}$, which is a severe suppression and will ensure that the field is pegged near $\phi=0$ for $T \gtrsim m_{\chi}^{i}$.

The above formula is valid when the DM is relativistic and in thermal equilibrium with the SM. However, we would like to keep $\phi \ll \phi_{0}$ until freeze-out. Since for the largest values of the final DM mass $m_{\chi}^{f} \sim 10^{9} \mathrm{GeV}$ considered here we have $m_{\chi}^{i} / m_{\chi}^{f} \sim 10^{-10}$, we would like to have $\phi\left(T_{f}\right) / \phi_{0} \lesssim m_{\chi}^{i} / m_{\chi}^{f}$, so that DM mass remains low enough throughout the freeze-out process. One can easily check that the discussion presented for this choice of parameters can be applied to other large values of $m_{\chi}^{f}$ considered in this work.

We note that $m_{\chi}^{f} \sim 10^{9} \mathrm{GeV}$ for the THUMP mass corresponds to a relic number density of $n_{\chi} \sim 10^{-18} T_{f}^{3}$ at freeze out, compared to $\sim T^{3}$ in the relativistic regime. The in-medium "tadpole" term $\lambda \phi \bar{\chi} \chi \rightarrow \lambda \phi n_{\chi} \sim 10^{-3} \mathrm{eV}^{3} \phi$ would then dominate the vacuum term $m_{\phi}^{2} \phi_{0} \phi \sim\left(10^{-4} \mathrm{eV}^{3}\right) \phi$. The in-medium "plasma frequency," given by $\omega_{\phi}^{2} \sim$ $\lambda^{2} n_{\chi} / m_{\chi}^{i} \sim 10^{-17} \mathrm{eV}^{2}$, is much larger than the vacuum parameter $m_{\phi}^{2} \sim 10^{-28} \mathrm{eV}^{2}$. Given the dominance of the medium effects at $T_{f} \sim 10 \mathrm{MeV}$, we note that the value of $\phi$ at this temperature is roughly given by $\phi\left(T_{f}\right) / \phi_{0} \sim m_{\chi}^{i} /\left(\lambda \phi_{0}\right) \sim m_{\chi}^{i} / m_{\chi}^{f}$, which implies that the value of $m_{\chi}$ is near its initial value all the way down to freeze out temperature and the relic number density calculations are governed by $m_{\chi}^{i}$, as assumed in our treatment.

The above discussion implicitly leads to the conclusion that due to the non-negligible coupling to DM, $\phi$ remains in thermal contact with the SM until temperatures near freezeout. In our analysis the smallest freeze-out temperature is $\sim 10 \mathrm{MeV}$ below which $\phi$ contributes to radiation in a similar way as neutrinos. Below temperatures associated with electron-positron annihilation $(T \sim 1 \mathrm{MeV})$, the contribution of $\phi$ to the effective number of neutrino species is given by $\Delta N_{\text {eff }}=4 / 7 \sim 0.6$. For larger freeze-out temperature, this number would be somewhat smaller and hence a typical prediction of our scenario is $\Delta N_{\text {eff }} \lesssim 0.6$. Interestingly, these values could potentially lead to easing of the tension in the Hubble parameter implied by local and early universe measurements [26-30].

The preceding analysis also implies that the value of $\phi$ remains frozen until the "tadpole" term becomes dominated by its vacuum value. For this to happen, $n_{\chi}$ must be diluted by the expansion of the Universe corresponding to a temperature $T_{*}$ at which $\left(T_{*} / T_{f}\right)^{3} \sim 1 / 10$, so that the vacuum tadpole starts to dominate. We note that this also suggests that the evolution of $\phi$ is not instantaneous and the aforementioned mismatch between the initial energy density $m_{\phi}^{2} \phi_{0}^{2}$ and the eventual DM energy content is remedied. This is achieved by the dilution of the relic density $n_{\chi}$ for $T \sim T_{*}$ at which $\phi$ starts to move away from $\phi\left(T_{f}\right)$. The above treatment only considers in-medium terms of linear and quadratic order in $\phi$. This truncation is justified since $\phi$ starts out very small due to thermal effects prior to freeze out, as indicated by eq. (3.2). Having illustrated that the mass of $\chi$ remains close to $m_{\chi}^{i}$ with our assumptions about the model parameters, we will next examine the annihilation processes that set the relic abundance of $\chi$.

The DM particle $\chi$ could give the correct relic abundance through the annihilation process given in figure 1 (plus $u$-channel). The annihilation cross-section can be expanded 
in powers of velocity and written as

$$
\frac{1}{2} \sigma_{\bar{\chi} \chi \rightarrow A^{\prime} A^{\prime}} v \approx a+b v^{2}+\mathcal{O}\left(v^{4}\right)
$$

Using the results of refs. [11, 31, 32], we find

$$
\begin{aligned}
a & =\frac{g_{D}^{4}}{8 \pi m_{\chi}^{i 2}} \frac{\left(1-r^{2}\right)^{3 / 2}}{\left(2-r^{2}\right)^{2}}, \\
b & =\frac{g_{D}^{4}}{192 \pi m_{\chi}^{i 2}} \frac{\sqrt{1-r^{2}}\left(17 r^{6}-36 r^{4}+28 r^{2}+24\right)}{\left(2-r^{2}\right)^{4}} .
\end{aligned}
$$

Here, $r=m_{A^{\prime}} / m_{\chi}^{i}$, and the $a$ and $b$ coefficients represent the $s$-wave and $p$-wave contributions respectively. For the values considered in this work, $a \gg b v^{2}$, making this annihilation process $s$-wave dominated. Expanding eq. (3.4) around $r$ and in the limit that $r \ll 1$, we get

$$
\left\langle\sigma_{\bar{\chi} \chi \rightarrow A^{\prime} A^{\prime}} v\right\rangle \approx \frac{g_{D}^{4}}{16 \pi m_{\chi}^{i 2}} \sqrt{1-r^{2}} .
$$

Though this is a hidden sector annihilation, the dark sector and SM are in thermal equilibrium in the early Universe. DM $\chi$ freezes out when it is non-relativistic at a temperature corresponding to $x=x_{f}$, which is given by

$$
x_{f}=\ln \left[\frac{c(c+2)}{4 \pi^{3}} \sqrt{\frac{45}{2}} \frac{g_{\chi}}{\sqrt{g_{*}}} m_{\chi}^{i} M_{\mathrm{Pl}} \frac{a+6 b / x_{f}}{\sqrt{x_{f}}\left(1-3 / 2 x_{f}\right)}\right] .
$$

Here we take $c \sim 0.5, g_{\chi}$ counts the internal degrees of freedom of $\chi$, which in our case is a Dirac fermion, and hence $g_{\chi}=4$ [25]. The quantity $g_{*}$ counts the relativistic degrees of freedom and is evaluated at freeze-out. After freeze-out, the relic energy density of DM is given by

$$
\Omega_{\chi} h^{2}=\frac{Y_{\infty} s_{0} h^{2}}{\rho_{c}} m_{\chi}^{f}
$$

where $s_{0}=2891.2 \mathrm{~cm}^{-3}$ is the late time entropy density of the Universe, $\rho_{c}$ is the critical density defined above and $m_{\chi}^{f}$ is the final mass of the DM after $\phi \rightarrow \phi_{0}$. The quantity $Y_{\infty}$ represents the late time comoving number density of DM which is given by

$$
Y_{\infty}^{-1}=\sqrt{\frac{\pi}{45}} \sqrt{g_{*}} m_{\chi}^{i} M_{\mathrm{Pl}} \frac{a+3 b / x_{f}}{x_{f}} .
$$

The observed value of the DM energy density is given by $\Omega_{o b} h^{2} \approx 0.12$ [28]. For any set of input parameters $\left\{g_{D}, m_{\chi}^{i}, m_{A^{\prime}}\right\}$, we can determine the $a$ and $b$ terms in eq. (3.4), $x_{f}$ using eq. (3.6), and $Y_{\infty}$ in eq. (3.8), which together yield $\Omega_{\chi} h^{2}$ in eq. (3.7).

On the left panel of figure 2, we plot the DM relic abundance in the $m_{\chi}^{f}$ vs. $m_{\chi}^{i}$ parameter space and in the $m_{\chi}^{i}$ vs. $x_{f}$ parameter space, on the right panel. We assume $m_{\chi}^{i}=2 m_{A^{\prime}}$ (in general, it is sufficient to assume $m_{\chi}^{i} \gtrsim m_{A^{\prime}}$ ), for two values of the dark sector coupling $\alpha_{D}=g_{D}^{2} / 4 \pi$. The solid (dashed) diagonal line corresponds to the observed relic abundance for $\alpha_{D}=1.0\left(\alpha_{D}=0.5\right)$. In this work, we limit the dark sector coupling to $\alpha_{D}=1.0 \mathrm{in}$ 

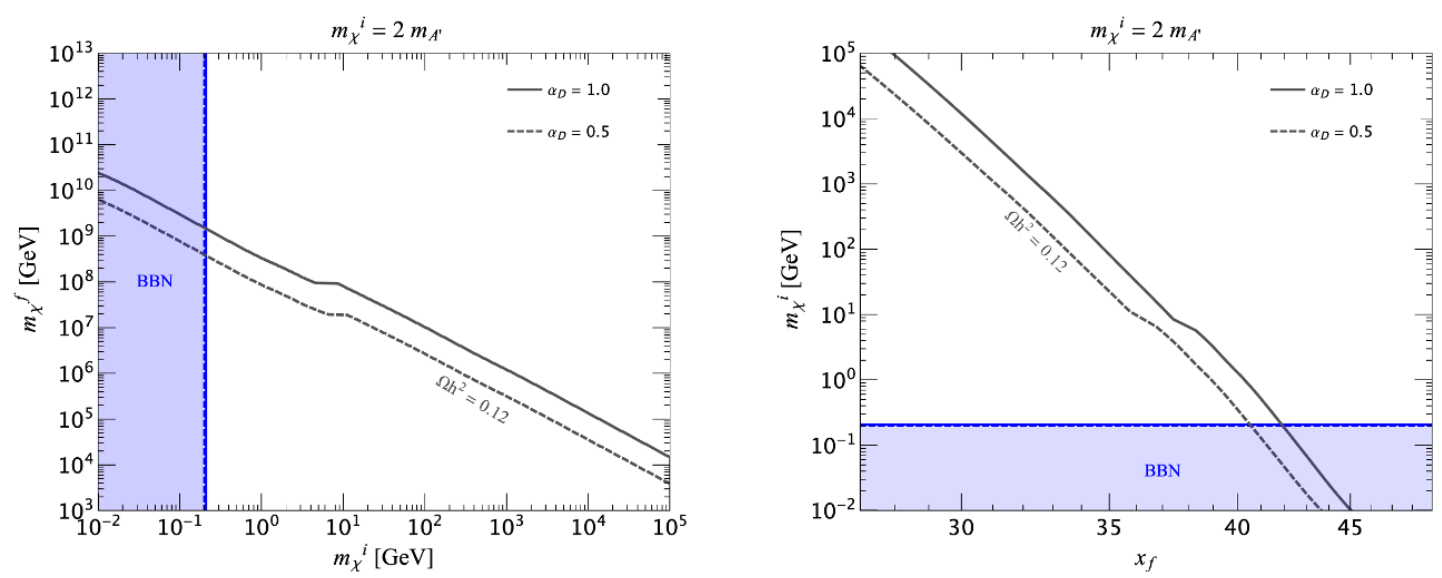

Figure 2. Relic abundance in the $m_{\chi}^{f}$ vs. $m_{\chi}^{i}$ parameter space on the left and in the $m_{\chi}^{i}$ vs. $x_{f}$ parameter space on the right. The solid diagonal line represents the thermal relic abundance corresponding to $\Omega_{\chi} h^{2} \approx \Omega_{o b} h^{2}[28]$ assuming $\alpha_{D}=1.0$ and the dashed line is one with $\alpha_{D}=0.5$. The blue shaded region represents the BBN bound on this model, in which any initial dark matter mass lower than $\sim 200 \mathrm{MeV}$ is excluded (see text for more details).

order to avoid our theory being non-perturbative, as we discussed above. In order to explain the observed relic abundance, we show that the maximum $m_{\chi}^{f}$ we can attain in this model is $\sim 10^{9} \mathrm{GeV}\left(\sim 10^{8} \mathrm{GeV}\right)$ for $\alpha_{D}=1.0\left(\alpha_{D}=0.5\right)$. Furthermore, we note that if we were to choose $\alpha_{D} \sim 4 \pi$ the maximum final mass we would obtain is $\sim 10^{11} \mathrm{GeV}$, though we do not consider this estimate to be reliable. In figure 2 , we see that near $m_{\chi}^{i} \sim 10 \mathrm{GeV}$ the relic abundance lines change shape; this is due to the fact $g_{*}(T)$ in our scenario is calculated at freeze-out, and the change in shape corresponds to the QCD phase transition in the early Universe. DM could annihilate during BBN, thus disrupting elemental abundances during this era. To be conservative, we assume that DM freezes out before BBN. The shaded blue region shows this BBN bound, which is independent of $m_{\chi}^{f}$, but dependent on $\alpha_{D}$ and $m_{\chi}^{i}$. To set this constraint, we assumed that $T_{\mathrm{BBN}} \sim 5 \mathrm{MeV}$ [33] and used eq. (3.6) to find the initial DM mass $m_{\chi}^{i}$ corresponding to freeze-out during BBN. For the benchmark values we have chosen here the boundary occurs near $m_{\chi}^{i} \sim 200 \mathrm{MeV}$, as shown by the solid (dashed) line corresponding to $\alpha_{D}=1.0\left(\alpha_{D}=0.5\right)$. The figure on the right panel can be translated to obtain the temperature at which DM freezes-out for a given $m_{\chi}^{i}$, since $x_{f}=m_{\chi}^{i} / T_{f}$.

\section{Constraints and phenomenology}

In this section we include the various experimental bounds that apply to our model space. In our Galactic neighborhood the virial DM velocity is $v \sim 10^{-3}$. If a DM particle of mass $m_{\chi}^{f}$ scatters with a nucleon of mass $m_{n} \sim 1 \mathrm{GeV}$, it will transfer momentum $q \sim m_{n} v \lesssim$ $1 \mathrm{MeV}$. Throughout this study, we assume $m_{A^{\prime}} \leq 0.5 m_{\chi}^{i}$ and in figure 2 , the largest allowed value of $m_{\chi}^{f} \sim 10^{9} \mathrm{GeV}$ corresponds to $m_{\chi}^{i} \sim 300 \mathrm{MeV}$. Hence for $m_{A^{\prime}} \sim 150 \mathrm{MeV}$, $m_{A^{\prime}} \gg q$ and the DM scattering cross-section is given by [34]

$$
\sigma_{n \chi} \approx \frac{16 \pi \mu_{n \chi}^{2} \varepsilon^{2} \alpha \alpha_{D}}{m_{A^{\prime}}^{4}},
$$




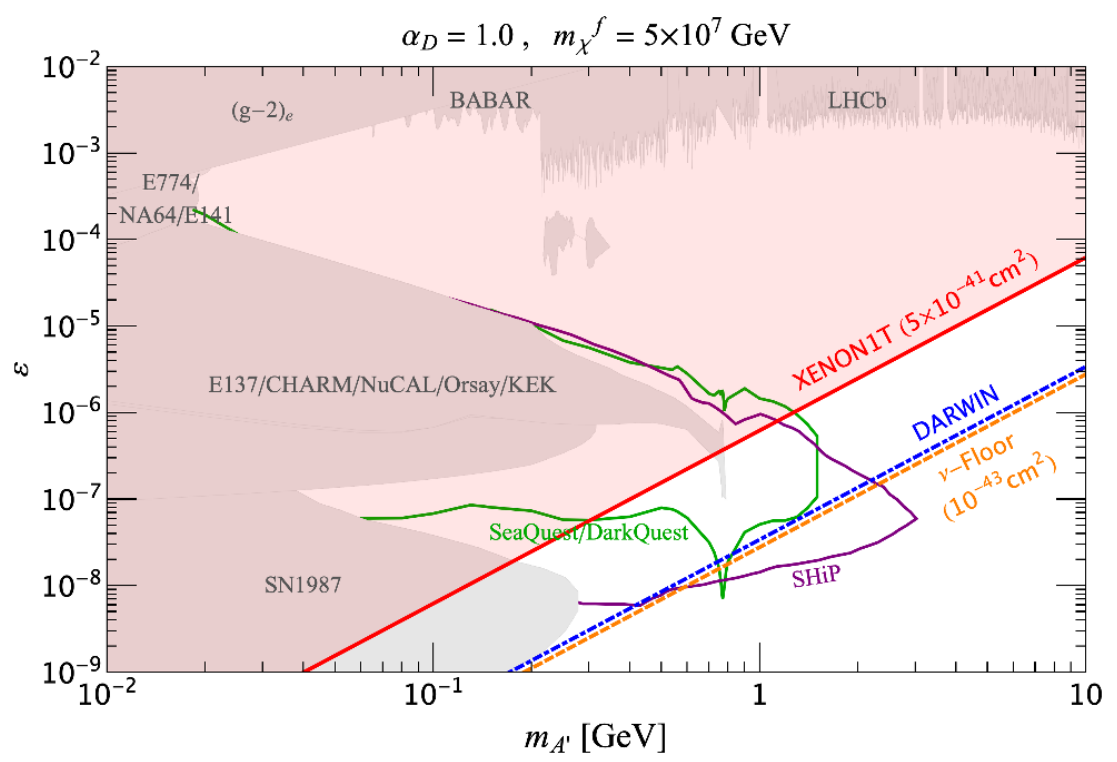

Figure 3. Constraints on the $\varepsilon$ vs. $m_{A^{\prime}}$ parameter space, taking $\alpha_{D}=1.0$ and $m_{\chi}^{f}=5 \times 10^{7} \mathrm{GeV}$. The grey shaded regions are the current bounds on a visibly decaying dark photon from various accelerator experiments and astrophysical measurements (see text for details). The red shaded region is ruled out by the latest run of the XENON1T experiment. The blue dot-dashed line is the projected sensitivity of the DARWIN experiment and the orange dashed line is the boundary of the neutrino background region for direct detection experiments. The projected sensitivity from the SeaQuest/DarkQuest and SHiP experiments are represented by the regions bounded by the green and purple lines respectively.

where $\mu_{n \chi}$ is the reduced DM-nucleon mass. For the DM masses considered here, the reduced mass to very good approximation is given by $\mu_{n \chi} \approx m_{n}$.

In figure 3 we show the current model constraints, in the $\varepsilon$ vs. $m_{A^{\prime}}$ parameter space, for benchmark $\alpha_{D}=1.0$ and $m_{\chi}^{f}=5 \times 10^{7} \mathrm{GeV}$. For the purposes of this study, we consider a mediator mass up to $10 \mathrm{GeV}$. Given our assumption that $m_{\chi}^{i}=2 m_{A^{\prime}}$, this corresponds to $m_{\chi}^{f}=5 \times 10^{7} \mathrm{GeV}$, which we choose as our benchmark in figure 3 . The grey shaded areas are current bounds on the visibly decaying dark photon from various fixed target and collider experiments ${ }^{2}$ [36-48], and from Supernova 1987A [49-51]. The most stringent direct-detection bounds on weak scale DM come from the XENON1T experiment and for $m_{\chi} \sim 1 \mathrm{TeV}$, the limit lies at $\sigma_{\chi n} \sim 10^{-45} \mathrm{~cm}^{2}$ [52]. The lack of a DM signal in XENON1T allows us to place a bound on our parameter space, which we obtain by rescaling the XENON1T limit with our mass of $m_{\chi}^{f}=5 \times 10^{7} \mathrm{GeV}$. This gives us a cross-section upper bound of $\sim 5 \times 10^{-41} \mathrm{~cm}^{2}$, which is represented by the red line in figure 3 . The red shaded region is ruled out by the XENON1T experiment. In a similar fashion, the blue dot-dashed line corresponds to $\sigma_{\chi n} \sim 1.5 \times 10^{-43} \mathrm{~cm}^{2}$ and is the projected sensitivity of the DARWIN experiment with a 200 ton-year exposure [53]. The orange dashed line is the boundary of the neutrino background, below which neutrinos start dominating the nuclear recoil spectrum [54].

\footnotetext{
${ }^{2}$ For the constraints not depicted here, see ref. [35].
} 
We also show projected sensitivity of future fixed target experiments. The region bounded by the purple solid line is the expected sensitivity reach for $\mathrm{SHiP}$ [48, 55], while the green solid line bounds the expected reach for SeaQuest/DarkQuest with $10^{20}$ protons on target $[48,56,57]$. For simplicity, here we only show projections from SeaQuest/DarkQuest and SHiP; for other experiments with similar reach, such as LongQuest or NA62, see ref. [48]. To obtain the bounds shown in grey, we used the publicly available DarkCast code [58] from ref. [35]. In figure 3, we show that future experiments such as SHiP or SeaQuest/DarkQuest would be able to probe the $\sim$ GeV-scale mediators of THUMP DM, corresponding to DM-nucleon cross-sections of $10^{-45}-10^{-40} \mathrm{~cm}^{2}$. Some of this parameter space may be probed by next generation direct detection experiments, such as DARWIN. However the $\mathrm{GeV}$-scale mediator signals available to accelerator experiments may provide hints of the dark sector below the neutrino floor.

We note that our model contains a long range force that acts on THUMPs with range typically of the order of $1 / m_{\phi} \sim 10^{4} \mathrm{~km}$. This could affect the distribution of DM on macroscopic scales with potentially observable effects. An examination of such effects is beyond the scope of this work. We also point out that a two-loop process mediated by DM and $A^{\prime}$ couples $\phi$ to ordinary charged matter, however this effect is too suppressed to have any observable effects.

\section{$5 \quad$ Discussion and conclusions}

In this work, we proposed a new mechanism for generating the thermal relic abundance of DM with masses above the so-called unitarity limit of $\sim 10^{2} \mathrm{TeV}$. We showed that DM would be composed of GeV-scale WIMPs in the early Universe, before decoupling from the thermal bath. If these WIMPs are coupled significantly to other lighter dark sector mediators, they could annihilate efficiently enough that the DM relic abundance after freeze-out is too small, given its initial mass, to constitute the observed DM energy density today. We postulate that the DM is coupled to an ultra-light scalar $\phi$ which is held near $\phi=0$ in its potential before the DM freezes out. After freeze-out, $\phi$ starts rolling to some large minimum, sourcing a large mass for the DM, thereby raising its energy density to the observed levels. We call the DM in our scenario a THermal Ultra Massive Particle or "THUMP."

In a specific model, we assumed that DM, which is initially at $\mathrm{GeV}$ scale can couple to a lighter dark photon mediator with strengths of up to $\alpha_{D}=1.0$. The strength of this coupling determines how low the DM number density can be after freeze-out, setting the maximum final THUMP mass that would be sourced by the scalar $\phi$ in order to get the correct relic abundance.

This model provides a connection between ultra heavy DM and GeV-scale mediators. Hence, our proposal motivates DM-nucleon cross-sections that are within reach of the next generation direct detection experiments as well as $\mathrm{GeV}$-scale mediators that can be probed at accelerators. Our scenario takes advantage of the various complementary methods of searching for DM, at both the cosmic and intensity frontiers. We further point out that the model discussed here may be extended to other examples such as a scalar mediator, which would give different phenomenology, however, we leave this for future work. 


\section{Acknowledgments}

We would like to thank David Curtin, Can Kilic, Michele Papucci, Arvind Rajaraman, Josh Ruderman, Tim Tait, Sean Tulin, Neal Weiner, and Yue Zhao for very helpful discussions. We would also like to thank Yu-Dai Tsai for very helpful correspondence regarding the dark photon bounds and projections. This work is supported by the United States Department of Energy under Grant Contract DE-SC0012704.

Open Access. This article is distributed under the terms of the Creative Commons Attribution License (CC-BY 4.0), which permits any use, distribution and reproduction in any medium, provided the original author(s) and source are credited.

\section{References}

[1] J. Preskill, M.B. Wise and F. Wilczek, Cosmology of the invisible axion, Phys. Lett. 120B (1983) 127 [INSPIRE].

[2] L.F. Abbott and P. Sikivie, A cosmological bound on the invisible axion, Phys. Lett. 120B (1983) 133 [INSPIRE].

[3] M. Dine and W. Fischler, The not so harmless axion, Phys. Lett. 120B (1983) 137 [INSPIRE].

[4] Particle Data Group collaboration, Review of particle physics, Chin. Phys. C 40 (2016) 100001 [INSPIRE].

[5] K. Griest and M. Kamionkowski, Unitarity limits on the mass and radius of dark matter particles, Phys. Rev. Lett. 64 (1990) 615 [InSPIRE].

[6] D.J.H. Chung, P. Crotty, E.W. Kolb and A. Riotto, On the gravitational production of superheavy dark matter, Phys. Rev. D 64 (2001) 043503 [hep-ph/0104100] [INSPIRE].

[7] K. Harigaya, T. Lin and H.K. Lou, GUTzilla dark matter, JHEP 09 (2016) 014 [arXiv: 1606.00923] [INSPIRE].

[8] K. Harigaya et al., Thermal relic dark matter beyond the unitarity limit, JHEP 08 (2016) 151 [arXiv: 1606.00159] [INSPIRE].

[9] E.W. Kolb and A.J. Long, Superheavy dark matter through Higgs portal operators, Phys. Rev. D 96 (2017) 103540 [arXiv: 1708.04293] [INSPIRE].

[10] A. Berlin, D. Hooper and G. Krnjaic, PeV-scale dark matter as a thermal relic of a decoupled sector, Phys. Lett. B 760 (2016) 106 [arXiv:1602.08490] [INSPIRE].

[11] A. Berlin, D. Hooper and G. Krnjaic, Thermal dark matter from a highly decoupled sector, Phys. Rev. D 94 (2016) 095019 [arXiv: 1609.02555] [InSPIRE].

[12] A. Berlin, WIMPs with GUTs: dark matter coannihilation with a lighter species, Phys. Rev. Lett. 119 (2017) 121801 [arXiv: 1704.08256] [INSPIRE].

[13] H. Kim and E. Kuflik, Superheavy thermal dark matter, Phys. Rev. Lett. 123 (2019) 191801 [arXiv: 1906.00981] [INSPIRE].

[14] J. Bramante, B. Broerman, R.F. Lang and N. Raj, Saturated overburden scattering and the multiscatter frontier: discovering dark matter at the Planck mass and beyond, Phys. Rev. D 98 (2018) 083516 [arXiv: 1803.08044] [INSPIRE]. 
[15] H. Davoudiasl and G. Mohlabeng, GeV-scale messengers of Planck-scale dark matter, Phys. Rev. D 98 (2018) 115035 [arXiv: 1809.07768] [InSPIRE].

[16] J. Bramante et al., Foraging for dark matter in large volume liquid scintillator neutrino detectors with multiscatter events, Phys. Rev. D 99 (2019) 083010 [arXiv:1812.09325] [INSPIRE].

[17] J. Bramante, J. Kumar and N. Raj, Dark matter astrometry at underground detectors with multiscatter events, Phys. Rev. D 100 (2019) 123016 [arXiv:1910.05380] [INSPIRE].

[18] L. Hui and E.D. Stewart, Superheavy dark matter from thermal inflation, Phys. Rev. D 60 (1999) 023518 [hep-ph/9812345] [INSPIRE].

[19] Y. Zhao, Cosmology and time dependent parameters induced by a misaligned light scalar, Phys. Rev. D 95 (2017) 115002 [arXiv:1701.02735] [INSPIRE].

[20] M.J. Baker, J. Kopp and A.J. Long, Filtered dark matter at a first order phase transition, arXiv: 1912.02830 [INSPIRE].

[21] L. Heurtier and H. Partouche, Spontaneous freeze out of dark matter from an early thermal phase transition, Phys. Rev. D 101 (2020) 043527 [arXiv:1912.02828] [InSPIRE].

[22] D. Chway, T.H. Jung and C.S. Shin, Dark matter filtering-out effect during a first-order phase transition, arXiv:1912.04238 [INSPIRE].

[23] M. Pospelov, A. Ritz and M.B. Voloshin, Secluded WIMP dark matter, Phys. Lett. B 662 (2008) 53 [arXiv: 0711.4866] [INSPIRE].

[24] B. Holdom, Two U(1)'s and epsilon charge shifts, Phys. Lett. 166B (1986) 196 [INSPIRE].

[25] E.W. Kolb and M.S. Turner, The early Universe, Front. Phys. 69 (1990) 1 [inSPIRE].

[26] J.L. Bernal, L. Verde and A.G. Riess, The trouble with $H_{0}$, JCAP 10 (2016) 019 [arXiv: 1607.05617] [INSPIRE].

[27] A.G. Riess et al., A 2.4\% determination of the local value of the Hubble constant, Astrophys. J. 826 (2016) 56 [arXiv: 1604.01424] [INSPIRE].

[28] Planck collaboration, Planck 2018 results. VI. Cosmological parameters, arXiv: 1807.06209 [INSPIRE].

[29] F. D'Eramo, R.Z. Ferreira, A. Notari and J.L. Bernal, Hot axions and the $H_{0}$ tension, JCAP 11 (2018) 014 [arXiv: 1808.07430] [INSPIRE].

[30] L.A. Anchordoqui and S.E. Perez Bergliaffa, Hot thermal universe endowed with massive dark vector fields and the Hubble tension, Phys. Rev. D 100 (2019) 123525 [arXiv: 1910.05860] [INSPIRE].

[31] J.M. Cline, G. Dupuis, Z. Liu and W. Xue, The windows for kinetically mixed Z'-mediated dark matter and the galactic center gamma ray excess, JHEP 08 (2014) 131 [arXiv: 1405.7691] [INSPIRE].

[32] M. Escudero, S.J. Witte and D. Hooper, Hidden sector dark matter and the Galactic center gamma-ray excess: a closer look, JCAP 11 (2017) 042 [arXiv: 1709.07002] [INSPIRE].

[33] H. Davoudiasl, D.E. Morrissey, K. Sigurdson and S. Tulin, Hylogenesis: a unified origin for baryonic visible matter and antibaryonic dark matter, Phys. Rev. Lett. 105 (2010) 211304 [arXiv: 1008.2399] [INSPIRE]. 
[34] R. Essig, J. Mardon and T. Volansky, Direct detection of sub-GeV dark matter, Phys. Rev. D 85 (2012) 076007 [arXiv: 1108.5383] [INSPIRE].

[35] P. Ilten, Y. Soreq, M. Williams and W. Xue, Serendipity in dark photon searches, JHEP 06 (2018) 004 [arXiv : 1801.04847] [INSPIRE].

[36] E.M. Riordan et al., A search for short lived axions in an electron beam dump experiment, Phys. Rev. Lett. 59 (1987) 755 [INSPIRE].

[37] J.D. Bjorken et al., Search for neutral metastable penetrating particles produced in the SLAC beam dump, Phys. Rev. D 38 (1988) 3375 [INSPIRE].

[38] NA48/2 collaboration, Search for the dark photon in $\pi^{0}$ decays, Phys. Lett. B 746 (2015) 178 [arXiv: 1504.00607] [INSPIRE].

[39] B. Batell, R. Essig and Z. Surujon, Strong constraints on sub-GeV dark sectors from SLAC beam dump E137, Phys. Rev. Lett. 113 (2014) 171802 [arXiv:1406.2698] [INSPIRE].

[40] H. Merkel et al., Search at the Mainz microtron for light massive gauge bosons relevant for the muon g- 2 anomaly, Phys. Rev. Lett. 112 (2014) 221802 [arXiv:1404.5502] [INSPIRE].

[41] KLOE-2 collaboration, Search for light vector boson production in $e^{+} e^{-} \rightarrow \mu^{+} \mu^{-} \gamma$ interactions with the KLOE experiment, Phys. Lett. B 736 (2014) 459 [arXiv:1404.7772] [INSPIRE].

[42] KLOE-2 collaboration, Limit on the production of a new vector boson in $\mathrm{e}^{+} \mathrm{e}^{-} \rightarrow \mathrm{U} \gamma$, $U \rightarrow \pi^{+} \pi^{-}$with the KLOE experiment, Phys. Lett. B 757 (2016) 356 [arXiv:1603.06086] [INSPIRE].

[43] NA64 collaboration, Search for invisible decays of sub-GeV dark photons in missing-energy events at the CERN SPS, Phys. Rev. Lett. 118 (2017) 011802 [arXiv:1610.02988] [INSPIRE].

[44] D. Hanneke, S.F. Hoogerheide and G. Gabrielse, Cavity control of a single-electron quantum cyclotron: measuring the electron magnetic moment, Phys. Rev. A 83 (2011) 052122 [arXiv: 1009.4831] [INSPIRE].

[45] BABAR collaboration, Search for a dark photon in $e^{+} e^{-}$collisions at BaBar, Phys. Rev. Lett. 113 (2014) 201801 [arXiv: 1406 . 2980] [INSPIRE].

[46] LHCb collaboration, Search for long-lived scalar particles in $B^{+} \rightarrow K^{+} \chi\left(\mu^{+} \mu^{-}\right)$decays, Phys. Rev. D 95 (2017) 071101 [arXiv:1612.07818] [INSPIRE].

[47] P. Ilten et al., Proposed inclusive dark photon search at LHCb, Phys. Rev. Lett. 116 (2016) 251803 [arXiv: 1603.08926] [INSPIRE].

[48] Y.-D. Tsai, P. deNiverville and M.X. Liu, The high-energy frontier of the intensity frontier: closing the dark photon, inelastic dark matter and muon $g-2$ windows, arXiv:1908.07525 [INSPIRE].

[49] J.H. Chang, R. Essig and S.D. McDermott, Revisiting supernova 1987A constraints on dark photons, JHEP 01 (2017) 107 [arXiv:1611.03864] [INSPIRE].

[50] J.H. Chang, R. Essig and S.D. McDermott, Supernova 1987A constraints on sub-GeV dark sectors, millicharged particles, the QCD axion and an axion-like particle, JHEP 09 (2018) 051 [arXiv: 1803.00993] [INSPIRE].

[51] D. Kazanas et al., Supernova bounds on the dark photon using its electromagnetic decay, Nucl. Phys. B 890 (2014) 17 [arXiv:1410.0221] [INSPIRE]. 
[52] XENON collaboration, Dark matter search results from a one ton-year exposure of XENON1T, Phys. Rev. Lett. 121 (2018) 111302 [arXiv:1805.12562] [INSPIRE].

[53] DARWIN collaboration, DARWIN: towards the ultimate dark matter detector, JCAP 11 (2016) 017 [arXiv : 1606.07001] [INSPIRE].

[54] J. Billard, L. Strigari and E. Figueroa-Feliciano, Implication of neutrino backgrounds on the reach of next generation dark matter direct detection experiments, Phys. Rev. D 89 (2014) 023524 [arXiv: 1307.5458] [INSPIRE].

[55] S. Alekhin et al., A facility to search for hidden particles at the CERN SPS: the SHiP physics case, Rept. Prog. Phys. 79 (2016) 124201 [arXiv: 1504.04855] [INSPIRE].

[56] S. Gardner, R.J. Holt and A.S. Tadepalli, New prospects in fixed target searches for dark forces with the SeaQuest experiment at Fermilab, Phys. Rev. D 93 (2016) 115015 [arXiv: 1509.00050] [INSPIRE].

[57] A. Berlin, S. Gori, P. Schuster and N. Toro, Dark sectors at the Fermilab SeaQuest experiment, Phys. Rev. D 98 (2018) 035011 [arXiv: 1804.00661] [INSPIRE].

[58] Darkcast code, https://gitlab.com/philten/darkcast (2019). 